\title{
THERMAL RADIATION, CHEMICAL REACTION, VISCOUS AND JOULE DISSIPATION EFFECTS ON MHD FLOW EMBEDDED IN A POROUS MEDIUM
}

\author{
B. ZIGTA \\ College of Natural and Computational Science, Debreberhan University \\ ETHIOPIA \\ E-mail: tzigta@yahoo.com
}

\begin{abstract}
An analysis is presented to study the effects of thermal radiation, chemical reaction, viscous and Joule dissipation on MHD free convection flow between a pair of infinite vertical Couette channel walls embedded in a porous medium. The fluid flows by a strong transverse magnetic field imposed perpendicularly to the channel wall on the assumption of a small magnetic Reynolds number. The governing non linear partial differential equations are transformed in to ordinary differential equations and are solved analytically. The effect of various parameters viz., Eckert number, electric conductivity, dynamic viscosity and strength of magnetic field on temperature profile has been discussed and presented graphically.
\end{abstract}

Key words: thermal radiation, chemical reaction, Joule dissipation, viscous dissipation, porous medium.

\section{Introduction}

Magnetohydrodynamics is the study of flow of an electrically conducting fluid combined with magnetic field. The phrase magnetohydrodynamics is derived from three Greek words. Magneto means magnetic field, Hydro means water, and dynamics means in motion. Hydrodynamics is the study of fluid flow and the forces, viz., inertial, thermal, buoyancy and viscous forces that cause the flow in the absence of electromagnetic field.

In fluid dynamics the word dissipation means conversion of energy from one form to other forms. In the viscous fluid flow, the velocity of the fluid takes energy from the motion of the fluid and transforms it into internal energy. This means the kinetic energy is dissipated, i.e., during the flow it is converted into internal energy. This process is irreversible in case of a viscous fluid and is known as viscous dissipation. Viscous dissipation acts as an energy source by changing the temperature distribution of the fluid which affects the heat transfer rate. The effect of viscous dissipation depends on whether the channel wall is cooled or heated.

Joule dissipation is the process by which the passage of an electric current through a conductor produces heat by affecting the whole electric conductor. In an MHD flow Joule dissipation acts as volumetric heat source.

In thermodynamics, the internal energy of the system is the energy contained within the system excluding the kinetic energy of the motion of the system as a whole and the potential energy of the system as a whole due to external force fields.

An unsteady Hartmann flow between two infinite parallel plates with viscous and Joule dissipation under an exponentially decaying pressure gradient has been studied in [1]. Couette flow which is an important phenomenon with respect to engineering applications involving shear-driven has been studied in [2]. The problem of an unsteady MHD Couette flow of a viscoelastic fluid under an exponentially decaying pressure gradient with suction and injection through the plates and viscous and Joule dissipations and obtained graphical representations of the velocity and temperature distributions using the finite difference method considered in [3].

The study of free convection flow is important in understanding natural circulation in geothermal reservoirs, problems involving the spread of pollution and other energy-related engineering applications. The steady hydromagnetic mixed convection flow in a vertical channel with symmetric and asymmetric wall 
heating conditions in the presence or absence of heat generation or absorption was studied in [4]. The effect of natural convection on an unsteady Couette flow has been investigated in [5]. The combined effect of natural convection and a uniform transverse magnetic field when the magnetic field is fixed relative to the plate or fluid has been discussed in [6].

Thermal radiation effects in fluid flow are significant in many industrial applications where high temperatures are involved. In engine combustion chambers, furnaces and power plants for gas cooled nuclear reactors; thermal radiation has a considerable effect on heat transfer. The unsteady natural convection hydromagnetic Couette flow between two infinite vertical plates in the presence of thermal radiation was studied in [7]. The effects of viscous and Joule dissipations using Galerkin finite element has been studied in [8]. The effect of MHD on a free convection oscillatory Couette flow when the temperature and concentration oscillate with time in the presence of the thermal radiation and chemical reaction has been studied in [9].

Flows of fluids through a porous medium are of main and principal interest because they occur frequently in nature. Such fluid flows have attracted the attention of many scholars due to their application in science and technology, viz., ground water flow, plasma studies, geophysics, geology, aerodynamics, geothermal reservoirs, thermal engineering, petroleum engineering and agricultural engineering. Many scholars have studied MHD free convective energy and concentration flow in a porous medium to examine flow of oil, water and natural gas. Free convective energy flow through a porous plate, in relation to the application of the areas mentioned was studied in [10]. Free convective flow through a porous medium between two vertical parallel plates is studied in [11]. Unsteady free convective MHD fluid flow past a vertical porous plate has been investigated in [12]. The effects of radiation on free convection over vertical flat plates embedded in a porous medium with high porosity are studied in [13]. The effect of radiation on chemically reacting MHD boundary layer flow of energy and concentration through a porous vertical flat plate is investigated in [14]. The effects of radiation on an unsteady MHD free convective flow past an oscillating vertical porous plate embedded in a porous medium with oscillatory heat flux are investigated in [15]. The effects of thermal radiation and magnetic field on an unsteady boundary layer mixed convection flow and heat transfer problem from a vertical porous stretching surface are studied in [16]. The simultaneous effects of opposing buoyancy force on heat and mass transfer by free convection in a fluid saturated by a porous medium are studied in [17].

The effect of MHD on a free convection oscillatory Couette flow when the temperature and concentration oscillate with time in the presence of thermal radiation and chemical reaction has been studied in [9]. In this study the effects of viscous and Joule dissipation has not been considered.

The main objective of this study is to analyze the effect of MHD flow when temperature and concentration fluctuate with time in the presence of thermal radiation, chemical reaction, viscous and Joule dissipation embedded in a porous channel walls.

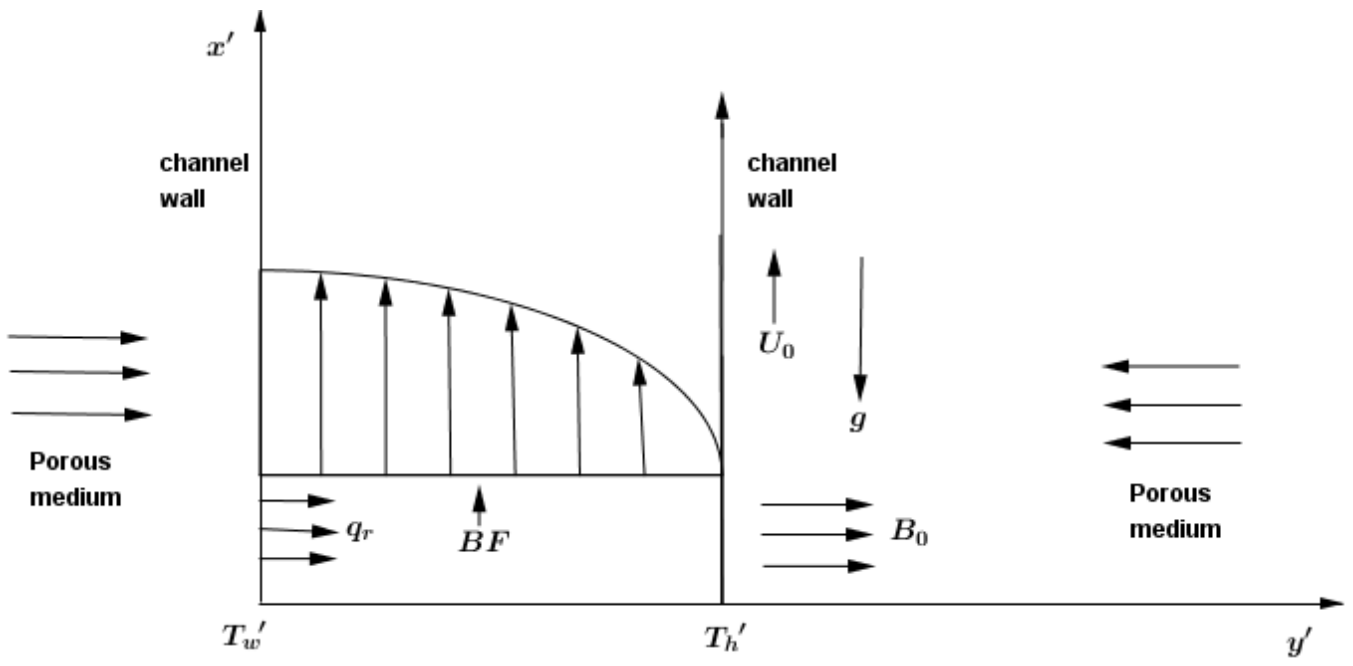

Fig.1. Flow configuration and coordinate system of the model. 


\section{Mathematical analysis}

Consider a two dimensional free convection Couette flow of an unsteady, incompressible, viscous, electrical conducting, Newtonian, chemical reacting and radiating fluid. The fluid flow is bounded by two infinite vertical channel walls separated by a distance $h$ embedded in a porous medium. Furthermore, the fluid is considered to be a gray in color, has radiation absorbing emitting nature but in a non-scattering medium in the optically thick limit. Roseland approximation is used to describe the radiative heat flux in the energy equation. It is also assumed that the radiation heat flux in the $x^{\prime}$ direction is negligible as compared to that in the $y^{\prime}$ direction.

The flow configuration and the coordinate system are shown in Fig.1. The $x^{\prime}$ axis is taken along the infinite channel walls and the $y^{\prime}$ axis is taken normal to the channel walls. The vertical moving channel wall is located at $y^{\prime}=0$ along the $x^{\prime}$ axis where the temperature is $T_{w}^{\prime}$ and the concentration is $C_{w}^{\prime}$. The other stationary channel wall is located at $y^{\prime}=h$ where the temperature is $T_{h}^{\prime}$ and the concentration is $C_{h}^{\prime}$.

Initially, at $t^{\prime}=0$, the stationary channel wall and the fluid are at the same temperature $T_{h}^{\prime}$ and the concentration level of the fluid $C_{h}^{\prime}$ is the same at all points. At a later time $t^{\prime}>0$ the temperature of the moving wall and concentration of the fluid increase $T_{w}^{\prime}$ and $C_{w}^{\prime}$ respectively and are maintained constant thereafter.

Free stream oscillates with time and has the form

$$
U^{\prime}\left(t^{\prime}\right)=U_{o}\left(1+\varepsilon e^{i \omega^{\prime} t^{\prime}}\right)
$$

In Eq.(2.1), $U_{0}$ is the mean constant free stream velocity, $\omega^{\prime}$ is the frequency and $t^{\prime}$ is the time.

To derive the governing equations of the model the following model assumptions are made:

(1) all fluid properties are constant except the influence of the density variation with temperature and concentration in the body force term,

(2) the Eckert number and magnetic Reynolds numbers are small so that the induced magnetic field of the fluid is negligible,

(3) the external electric field is zero and the electric field due to the polarization of charges is negligible,

(4) viscosity is also considered with the constant permeability of the porous medium,

(5) there exists a homogeneous chemical reaction of first order with a constant rate between diffusing concentration and the fluid in the moving plate,

(6) a uniform magnetic field is applied in the direction perpendicular to the channel walls,

(7) all the physical variables are independent of $x^{\prime}$.

Based on the model assumptions, the governing equation has the following form.

$$
\frac{\partial u^{\prime}}{\partial t^{\prime}}=\frac{\partial U^{\prime}}{\partial t^{\prime}}+v\left(\frac{\partial^{2} u^{\prime}}{\partial y^{\prime 2}}\right)+g \beta\left(T^{\prime}-T_{h}^{\prime}\right)+g \beta_{c}\left(C^{\prime}-C_{h}^{\prime}\right)-\left(\frac{J X B}{\rho}\right)-\frac{v u^{\prime}}{k^{\prime}}
$$

In Eq.(2.2), the vector cross product $J x B$ represents the Lorentz force. This term is a body force corresponding to magnetohydrodynamics flow. The total magnetic field is represented by $B$. The density of the current is represented by $J$ and $k^{\prime}$ denotes the permeability of the porous medium. The minus sign in the sixth term of the right hand side indicates that the fluid flows from a higher to a lower potential.

Also, the expression for Lorentz force reduces and takes the form as 


$$
J x B=-\sigma B^{2} u^{\prime} .
$$

In view of the result Eq.(2.3), Eq.(2.2) reduces to

$$
\frac{\partial u^{\prime}}{\partial t^{\prime}}=\frac{\partial U^{\prime}}{\partial t^{\prime}}+v \frac{\partial^{2} u^{\prime}}{\partial y^{\prime 2}}+g \beta\left(T^{\prime}-T_{h}^{\prime}\right)+g \beta_{c}\left(C^{\prime}-C_{h}^{\prime}\right)-\frac{\sigma B^{2}\left(u^{\prime}-U^{\prime}\right)}{\rho}-\frac{v u^{\prime}}{k^{\prime}}
$$

The energy equation of the model can be expressed as

$$
\frac{\partial T^{\prime}}{\partial t^{\prime}}=\alpha\left(\frac{\partial^{2} T^{\prime}}{\partial y^{\prime 2}}\right)-\left(\frac{1}{\rho C_{p}}\right)\left(\frac{\partial q_{r}}{\partial y^{\prime}}\right)+\left(\frac{\mu}{\rho C_{p}}\right)\left(\frac{\partial u^{\prime}}{\partial y^{\prime}}\right)^{2}+\frac{\sigma B^{2} u^{\prime 2}}{\rho C_{p}} .
$$

The concentration equation of the model can be expressed as

$$
\frac{\partial C^{\prime}}{\partial t^{\prime}}=D\left(\frac{\partial^{2} C^{\prime}}{\partial y^{\prime 2}}\right)-K_{r}\left(C^{\prime}-C_{h}^{\prime}\right)
$$

Equations (2.4) - (2.6) govern the present model. Here $\alpha$ - is thermal diffusivity, $\rho$ - the density of the fluid, $g-$ the acceleration due to gravity, $\beta$ - the thermal expansion coefficient, $\beta_{c}-$ the concentration expansion coefficient, $T^{\prime}$-the temperature of the fluid in the boundary layer, $T^{\prime}{ }_{w}-$ the temperature of moving channel wall, $T_{h}^{\prime}$-the temperature of stationary channel wall, $C_{p}$ - the specific heat capacity at constant pressure, $\mu$-the dynamic viscosity, $\sigma$-electric conductivity, $q_{r}$-the local radiative heat flux, $B$ - the magnetic induction, $C^{\prime}$-the concentration of the fluid in the boundary layer, $C_{w}^{\prime}$ - the concentration of the moving channel wall, $C_{h}^{\prime}$-the concentration of the stationary channel wall, $D$ - the molecular diffusivity, $K_{r}$ - the chemical reaction parameter.

Since the free stream velocity, temperature and concentration fluctuates with time the boundary conditions of the model have the form

$$
\begin{aligned}
& y^{\prime}=0, \quad u^{\prime}=U_{o}\left(1+\varepsilon e^{i \omega^{\prime} t^{\prime}}\right), \\
& T^{\prime}=T_{w}^{\prime}+\varepsilon\left(T_{w}^{\prime}-T_{h}^{\prime}\right) e^{i \omega^{\prime} t^{\prime}}, \\
& C^{\prime}=C_{w}^{\prime}+\varepsilon\left(C_{w}^{\prime}-C_{h}^{\prime}\right) e^{i \omega^{\prime} t^{\prime},} \\
& y^{\prime}=b, \quad u^{\prime}=0, \quad T^{\prime}=T_{h}^{\prime}, \quad C^{\prime}=C_{h}^{\prime} .
\end{aligned}
$$

The Roseland approximation for radiative heat flux [18] is given by

$$
q_{r}=\left(\frac{-4 \sigma}{3 k_{s}}\right)\left(\frac{\partial T^{1^{4}}}{\partial y^{\prime}}\right)
$$


In Eq.(2.11), the parameters $\sigma$ and $k_{s}$ represent the Stefan Boltzmann constant and the Roseland mean absorption coefficient, respectively.

Taking the Taylor series expansion of $T^{14}$ and neglecting terms with higher powers, we have

$$
T^{\prime 4} \cong 4 T_{h}^{\prime 3} T^{\prime}-3 T_{h}^{\prime 4}
$$

In view of Eqs (2.11) and (2.12), Eq.(2.4) reduces to

$$
\frac{\partial T^{\prime}}{\partial t^{\prime}}=\left(\frac{1}{\rho C_{p}}\right)\left[\kappa+\left(\frac{16 \sigma T_{h}^{\prime 3}}{3 k_{s}}\right)\right]\left(\frac{\partial^{2} T^{\prime}}{\partial y^{\prime 2}}\right)+\left(\frac{Q_{0}}{\rho C_{p}}\right)\left(T^{\prime}-T_{h}^{\prime}\right)+\left(\frac{\mu}{\rho C_{p}}\right)\left(\frac{\partial u^{\prime}}{\partial y^{\prime}}\right)^{2}+\frac{\sigma B^{2} u^{\prime 2}}{\rho C_{p}} .
$$

In Eq.(2.13) the third and fourth term denote viscous and Joule dissipation, respectively.

\section{Non dimensionalization of the model} quantities

The dimensionless form of the model is found by introducing the following non-dimensional

$$
\begin{aligned}
& y=y^{\prime} / h ; \quad R=4 \sigma T_{h}^{\prime 3} / \kappa k_{s} ; \quad u=u^{\prime} / U_{0} ; \quad U=U^{\prime} / U_{0} ; \quad t=\omega^{\prime} t^{\prime} ; \quad \omega=\omega^{\prime} h^{2} / v ; \\
& \mathrm{Gc}=\left[\left[g \beta_{c} h^{2}\left(C_{w}^{\prime}-C_{h}^{\prime}\right)\right] / v U_{0}\right] ; \quad \mathrm{Gr}=\left[g \beta h^{2}\left(T_{w}^{\prime}-T_{h}^{\prime}\right) / v U_{0}\right] ; \quad \mathrm{Sc}=v / D ; \quad \operatorname{Pr}=v / \alpha ; \\
& M=B h \sqrt{\sigma / \mu} ; \quad C=\left[\left(C^{\prime}-C_{h}^{\prime}\right) /\left(C_{w}^{\prime}-C_{h}^{\prime}\right)\right] ; \quad \mathrm{Ec}=U_{o}^{2} / C_{p} \Delta T ; \quad \Delta T=T_{w}^{\prime}-T_{h}^{\prime} ; \\
& \theta=\left[\left(T^{\prime}-T_{h}^{\prime}\right) /\left(T_{w}^{\prime}-T_{h}^{\prime}\right)\right] ; \quad K=k^{\prime} U_{0}^{2} / v^{2} ; \quad Q_{0}=16 \sigma T_{h}^{\prime 2} / k_{s} .
\end{aligned}
$$

Substituting the non-dimensional quantities in Eqs (2.4) - (2.6) the non-dimensional form of the model takes the following form

$$
\begin{aligned}
& \omega \frac{\partial u}{\partial t}=\omega \frac{\partial U}{\partial t}+\frac{\partial^{2} u}{\partial y^{2}}+\operatorname{Gr} \theta+\operatorname{Gc} C-M^{2}(u-U)-\frac{U_{0}^{2} u b^{2}}{K v^{2}} \\
& \omega \frac{\partial \theta}{\partial t}=\frac{1}{\operatorname{Pr}}\left(1+\frac{4 R}{3}\right) \frac{\partial^{2} \theta}{\partial y^{2}}+\frac{Q_{o}}{\rho C_{p} v} \theta h^{2}+\operatorname{Ec}\left(\frac{\partial u}{\partial y}\right)^{2}+\frac{\sigma B^{2} u^{2} U_{o} \beta g}{\mu C_{p} \mathrm{Gr}} \\
& \omega \mathrm{Sc} \frac{\partial C}{\partial t}=\frac{\partial^{2} C}{\partial y^{2}}-\frac{K_{r} C b^{2}}{D}
\end{aligned}
$$

The corresponding non dimensional boundary conditions (2.7)-(2.10) take the form

$$
\begin{aligned}
& y=0, \quad u=1+\varepsilon e^{i t}, \quad \theta=1+\varepsilon e^{i t}, \quad C=1+\varepsilon e^{i t}, \\
& y=1, \quad u=0, \quad \theta=0, \quad C=0 .
\end{aligned}
$$


The system of Eqs (3.1)-(3.3) together with the boundary conditions (3.4)-(3.5) constitutes the nondimensional form of the present model.

\section{Method of solution to the model problem}

When the amplitude of oscillations $(\varepsilon \ll 1)$ is very small we can assume the solutions of flow velocity $u$, temperature field $\theta$ and concentration $C$ near the moving plate as the sum of steady and small oscillating components.

Using the perturbation technique the solution of the model has the following form

$$
\begin{aligned}
& u(y, t)=u_{0}(y)+\varepsilon u_{1}(y) e^{i t}, \\
& \theta(y, t)=\theta_{0}(y)+\varepsilon \theta_{l}(y) e^{i t}, \\
& C(y, t)=C_{0}(y)+\varepsilon C_{l}(y) e^{i t} .
\end{aligned}
$$

In Eqs (4.1)-(4.3) $u_{0}, \theta_{0}$ and $C_{0}$ represent mean velocity, mean temperature and mean concentration respectively.

Also, the non dimensional free stream velocity takes the form

$$
U=1+\varepsilon e^{i t}
$$

Substituting Eqs (4.1)-(4.4) into Eqs (3.1)-(3.3), equating harmonic and non-harmonic terms and neglecting higher orders of $\varepsilon$ the following system of equations is obtained

$$
\begin{aligned}
& u_{0}{ }^{\prime \prime}-M^{2} u_{0}=-\mathrm{Gr} \theta_{0}-\mathrm{Gc}_{0}-M^{2}, \\
& u_{1}{ }^{\prime \prime}-\left(i \omega+M^{2}\right) u_{1}=-\mathrm{Gr} \theta_{1}-\mathrm{Gc} C_{1}-\left(i \omega+M^{2}\right), \\
& C_{0}{ }^{\prime}-\frac{K_{r} C_{o} h}{D}=0, \\
& C_{1}{ }^{\prime}-C_{1}\left(\frac{K_{r} h^{2}}{D}+i \omega \mathrm{Sc}\right)=0, \\
& \frac{1}{\operatorname{Pr}}\left(1+\frac{4 R}{3}\right) \theta_{0}{ }^{\prime}+\frac{Q_{0} \theta_{0} h^{2}}{\rho C_{p} v}+\frac{\sigma B^{2} u_{0}{ }^{2} U_{o} \beta g}{\mu C_{p} \mathrm{Gr}}=-\operatorname{Ec} u_{0}^{\prime}, \\
& \frac{1}{\operatorname{Pr}}\left(1+\frac{4 R}{3}\right) \theta_{1}{ }^{\prime \prime}+\left(\frac{Q_{0} h^{2}}{\rho C_{p} v}-i \omega\right) \theta_{1}+\frac{2 \sigma B^{2} u_{0} u_{l} U_{o} \beta g}{\mu C_{p} \mathrm{Gr}}=-2 \mathrm{Ec} u_{0}^{\prime} u_{1}^{\prime} .
\end{aligned}
$$

Further, the new boundary conditions corresponding to Eqs (3.4)-(3.5) are obtained as

$$
y=0, \quad u_{0}=1, \quad u_{1}=1, \quad \theta_{0}=1, \quad \theta_{1}=1, \quad C_{0}=1, \quad C_{1}=1,
$$




$$
y=1, \quad u_{0}=0, \quad u_{1}=0, \quad \theta_{0}=0, \quad \theta_{1}=0, \quad C_{0}=0, \quad C_{1}=0 .
$$

The variables $u_{0}, u_{1}, \theta_{0}, \theta_{1}, C_{0}$ and $C_{1}$ are still coupled in Eqs (4.5)-(4.10). To solve Eqs (4.5)-(4.10) it is assumed that heat due to viscous dissipation is superimposed on the motion. Mathematically, this can be expressed by expanding the velocity, temperature and concentration terms in power of Ec. In the case of incompressible fluids, Ec is always very small and we assume that

$$
W(y)=W_{0}(y)+\operatorname{Ec} W_{l}(y)+o\left(\mathrm{Ec}^{2}\right)
$$

Here $W$ stands for any variable $u_{0}, u_{1}, \theta_{0}, \theta_{1}, C_{0}$ and $C_{1}$. These variables can be expanded in powers of Ec as follows

$$
\begin{aligned}
& u_{0}(y)=u_{00}(y)+\operatorname{Ec} u_{01}(y), \\
& u_{1}(y)=u_{10}(y)+\operatorname{Ec} u_{11}(y), \\
& \theta_{0}(y)=\theta_{00}(y)+\operatorname{Ec} \theta_{01}(y), \\
& \theta_{1}(y)=\theta_{10}(y)+\operatorname{Ec} \theta_{11}(y), \\
& C_{0}(y)=C_{00}(y)+\operatorname{Ec} C_{01}(y), \\
& C_{1}(y)=C_{10}(y)+\operatorname{Ec} C_{11}(y) .
\end{aligned}
$$

Upon substituting Eqs (4.13)-(4.18) into Eqs (4.5)-(4.10) and equating terms free from Ec and with coefficients Ec and neglecting higher orders of Ec the following equations are obtained

$$
\begin{aligned}
& u{ }_{00}-M^{2} u_{00}=-\operatorname{Gr} \theta_{00}-\operatorname{Gc} C_{00}-M^{2}, \\
& u^{\prime \prime}{ }_{01}-M^{2} u_{01}=-\operatorname{Gr} \theta_{01}-\operatorname{Gc} C_{01}, \\
& u_{10}^{\prime \prime}-u_{10}\left(i \omega+M^{2}\right)=-\operatorname{Gr} \theta_{10}-\operatorname{Gc} C_{10}, \\
& u^{\prime \prime}{ }_{11}-u_{11}\left(i \omega+M^{2}\right)=-\operatorname{Gr} \theta_{11}-\operatorname{Gc} C_{11}, \\
& C{ }_{00}-\frac{k_{r} C_{00} h^{2}}{D}=0, \\
& C{ }_{01}-\frac{k_{r} C_{01} h^{2}}{D}=0, \\
& C{ }_{10}-C_{10}\left[\frac{k_{r} h^{2}}{D}+i \omega \mathrm{Sc}\right]=0,
\end{aligned}
$$




$$
\begin{aligned}
& C^{\prime \prime}{ }_{11}-C_{11}\left[\frac{k_{r} h^{2}}{D}+i \omega \mathrm{Sc}\right]=0, \\
& \frac{1}{\operatorname{Pr}}\left(1+\frac{4 R}{3}\right) \theta^{\prime \prime}{ }_{00}+\frac{Q_{0} \theta_{00} h^{2}}{\rho C_{p} v}+\frac{\sigma B^{2} u_{00}{ }^{2} U_{0} \beta g}{\mu C_{p} \mathrm{Gr}}=0, \\
& \frac{1}{\operatorname{Pr}}\left(1+\frac{4 R}{3}\right) \theta^{\prime \prime}{ }_{01}+\frac{Q_{0} \theta_{01} h^{2}}{\rho C_{p} v}+\frac{\sigma B^{2} u_{00} u_{01} U_{0} \beta g}{\mu C_{p} \mathrm{Gr}}=-u_{00}^{\prime}{ }^{2}, \\
& \frac{1}{\operatorname{Pr}}\left(1+\frac{4 R}{3}\right) \theta^{\prime \prime}{ }_{10}+\left[\frac{Q_{0} h^{2}}{\rho C_{p} v}-i \omega\right] \theta_{10}+\frac{2 \sigma B^{2} u_{00} u_{10} U_{0} \beta g}{\mu C_{p} \mathrm{Gr}}=0, \\
& \frac{1}{\operatorname{Pr}}\left(1+\frac{4 R}{3}\right) \theta^{\prime \prime}{ }_{11}+\left[\frac{Q_{0} h^{2}}{\rho C_{p} v}-i \omega\right] \theta_{11}+\frac{2 \sigma B^{2} U_{0} \beta g\left(u_{01} u_{10}+u_{00} u_{11}\right)}{\mu C_{p} \mathrm{Gr}}=-2 u^{\prime}{ }_{00} u_{10}^{\prime},
\end{aligned}
$$

Equations (4.19)-(4.30) are subjected to the new boundary conditions as given below

$$
\begin{aligned}
& y=0, \quad u_{00}=u_{10}=\theta_{00}=\theta_{10}=C_{00}=C_{10}=1, \quad u_{01}=u_{11}=\theta_{01}=\theta_{11}=C_{01}=C_{11}=0, \\
& y=1, \quad u_{00}=u_{01}=u_{10}=u_{11}=\theta_{00}=\theta_{01}=\theta_{10}=\theta_{11}=C_{00}=C_{01}=C_{10}=C_{11}=0 .
\end{aligned}
$$

Upon solving Eqs (4.19)-(4.30) together with the boundary conditions (4.31)-(4.32), the analytical solutions are obtained

$$
\begin{aligned}
& u_{00}(y)=A e^{M y}+B e^{-M y}+\frac{\mathrm{Gr} \theta_{00}+\mathrm{GcC}_{00}}{M^{2}}+1, \\
& u_{01}(y)=A^{\prime} e^{M y}+B^{\prime} e^{-M y}+\frac{\mathrm{Gr}_{01}+\mathrm{Gc} C_{01}}{M^{2}}=0, \\
& u_{10}(y)=c e^{\beta y}+d e^{-\beta y}+\frac{\mathrm{Gr}_{10}+\mathrm{Gc} C_{10}}{M^{2}}, \\
& u_{11}(y)=c^{\prime} e^{\beta y}+d^{\prime} e^{-\beta y}+\frac{\mathrm{Gr} \theta_{11}+\mathrm{Gc} C_{11}}{M^{2}}=0, \\
& \theta_{00}(y)=c_{1} e^{\lambda y}+c_{2} e^{-\lambda y}-\frac{\sigma B^{2} u_{00}{ }^{2} U_{0} \beta g(3+4 R)}{3 Q_{0} h^{2} \operatorname{Pr} \mathrm{Gr}}, \\
& \theta_{01}(y)=c_{1}^{\prime} e^{\lambda y}+c_{2}^{\prime} e^{-\lambda y}=0,
\end{aligned}
$$




$$
\begin{aligned}
& \theta_{10}(y)=k_{1} e^{\mu y}+k_{2} e^{-\mu y}-\frac{\sigma B^{2} u_{00} u_{10} U_{0} \beta g(3+4 R)}{3 \operatorname{Pr} \operatorname{Gr}\left(Q_{0} h^{2}-i \mu C_{p} \omega\right)}, \\
& \theta_{11}(y)=k_{1}^{\prime} e^{\mu y}+k_{2}^{\prime} e^{-\mu y}=0, \\
& C_{00}(y)=d_{1} e^{m y}+d_{2} e^{-m y}, \\
& C_{01}(y)=f_{1} e^{m y}+f_{2} e^{-m y}=0, \\
& C_{10}(y)=h_{1} e^{\gamma y}+h_{2} e^{-\gamma y}, \\
& C_{11}(y)=p_{1} e^{\gamma y}+p_{2} e^{-\gamma y}=0 .
\end{aligned}
$$

In Eqs (4.33)-(4.44), we used the following notations

$$
\begin{aligned}
& m= \pm \sqrt{\frac{k_{r} h^{2}}{D}}, \quad \gamma= \pm \sqrt{\frac{k_{r} h^{2}}{D}+i \omega \mathrm{Sc}}, \quad \lambda= \pm i \sqrt{3 Q_{0} h^{2} \operatorname{Pr} / \rho C_{p} v(4 R+3)}, \\
& c=\frac{-1}{e^{2 \beta}-1}, \quad d=\frac{e^{2 \beta}}{e^{2 \beta}-1}, \quad A=\frac{M^{2}-(\mathrm{Gr}+\mathrm{Gc}) e^{-M}}{M^{2}\left(e^{-M}-e^{M}\right)}, \quad B=\frac{M^{2}-(\mathrm{Gr}+\mathrm{Gc}) e^{M}}{M^{2}\left(e^{M}-e^{-M}\right)}, \\
& \beta= \pm \sqrt{i \omega+M^{2}}, \quad \mu= \pm i \sqrt{3 \operatorname{Pr}\left(Q_{0} h^{2}-i \omega \rho C_{p} v\right) / \rho C_{p} v(4 R+3)}, \\
& c_{1}=\frac{1}{e^{2 \lambda}-1}, \quad c_{2}=\frac{e^{2 \lambda}}{e^{2 \lambda}-1}, \quad d_{1}=\frac{-1}{e^{2 m}-1}, \quad d_{2}=\frac{e^{2 m}}{e^{2 m}-1}, \quad h_{1}=\frac{2-e^{2 \gamma}}{1-e^{2 \gamma}}, \quad h_{2}=\frac{-1}{1-e^{2 \gamma}}, \\
& k_{1}=\frac{-1}{e^{2 \mu}-1}, \quad k_{2}=\frac{e^{2 \mu}}{e^{2 \mu}-1} .
\end{aligned}
$$

\section{Results and discussion of simulation study}

In this paper, the effect of thermal radiation, chemical reaction, viscous and Joule dissipation on MHD free convection flow in a porous vertical channel walls has been studied. The effect of physical parameters such as Eckert number, electric conductivity, strength magnetic field and dynamic viscosity on temperature has been analyzed. Perturbation technique is used to convert the governing non linear partial differential equations to a system of ordinary differential equations which can be solved analytically. MATLAB and GeoGebra software are used to draw the graphs.

For the sake of brevity only the graphical representation of the temperature profile for different values of the Eckert number, electric conductivity, strength of magnetic field and dynamic viscosity presented. The other parameters such as the Grashof number, i.e. based on temperature difference, the modified Grashof number, i.e. based on concentration difference, radiation parameter, chemical reaction parameter, the Prandtl number, Schmidt number and molecular diffusivity have not been discussed. 
In Fig.2 the influence of the Eckert number on temperature of the fluid has been presented. The graph of temperature versus distance for different values of the Eckert number has been drawn. From the simulated graph it can be concluded that as the Eckert number increases the temperature decreases. Physically, this shows how kinetic energy can be changed to internal energy by deformation of fluid elements.

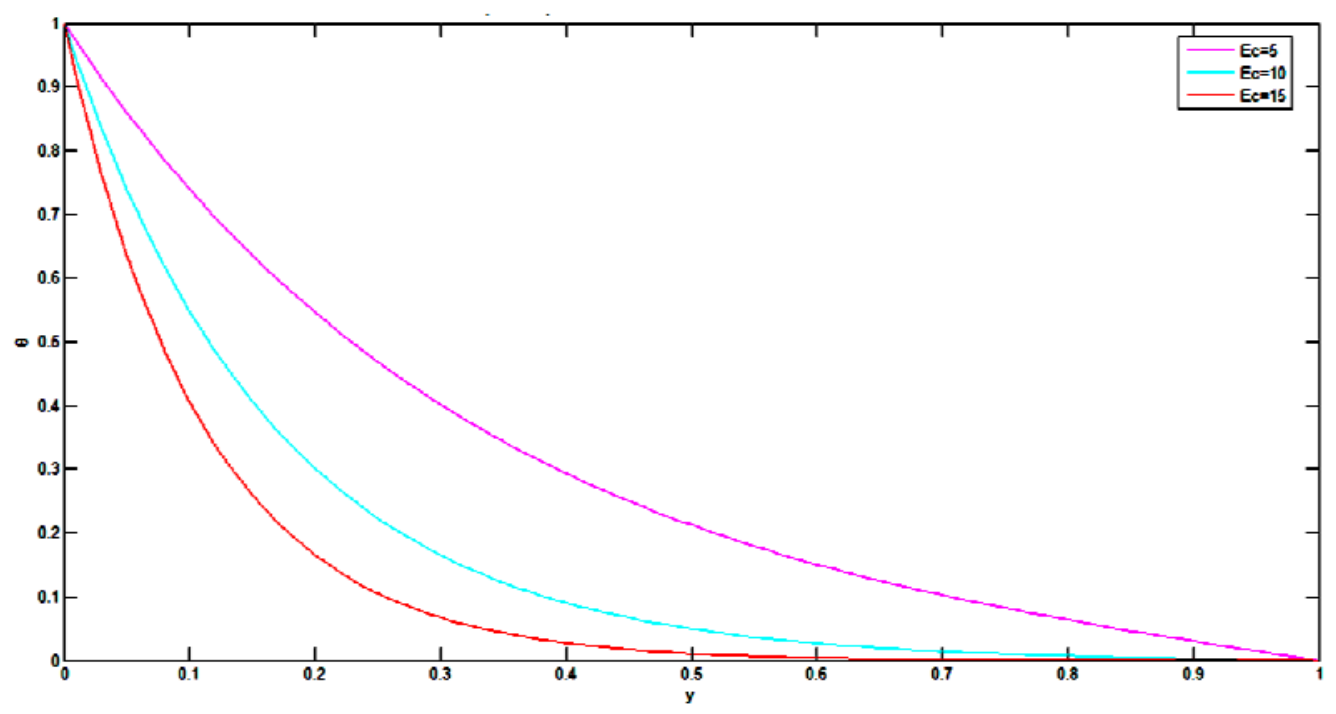

Fig.2. Temperature profile of the model problem for different values of the Eckert number.

Figure 3 illustrates the influence of electric conductivity on temperature of the fluid. The graph is plotted yversus $\theta$ representing respectively the distance between the channel walls and temperature. It can be observed from the graph that as electrical conductivity increases the temperature decreases.

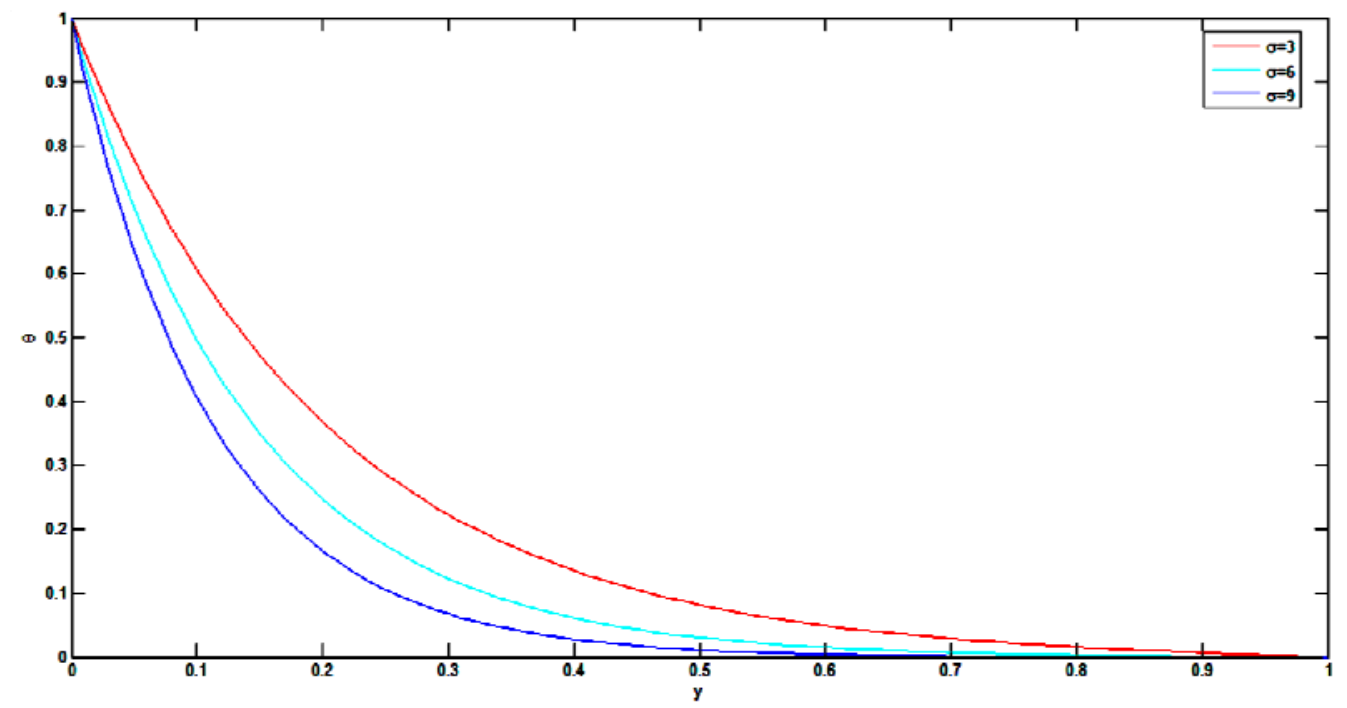

Fig.3. Temperature profile of the model for different values of electric conductivity.

In Fig.4 the simulated results of the influence of the strength of magnetic field on temperature profile has been investigated. The graph is drawn $y$ versus $\theta$ where $y$ denotes the distance between the channel walls while $\theta$ represents temperature of the fluid. From the results of the simulated graph it can be observed that as the strength of magnetic field increases the temperature of the fluid decreases. This is due to the 
Lorentz force which resists the fluid flow. Furthermore, the increment of the strength of magnetic field results in a decrement of both temperature and velocity of the fluid. The former theoretical fact has been verified by the simulation study.

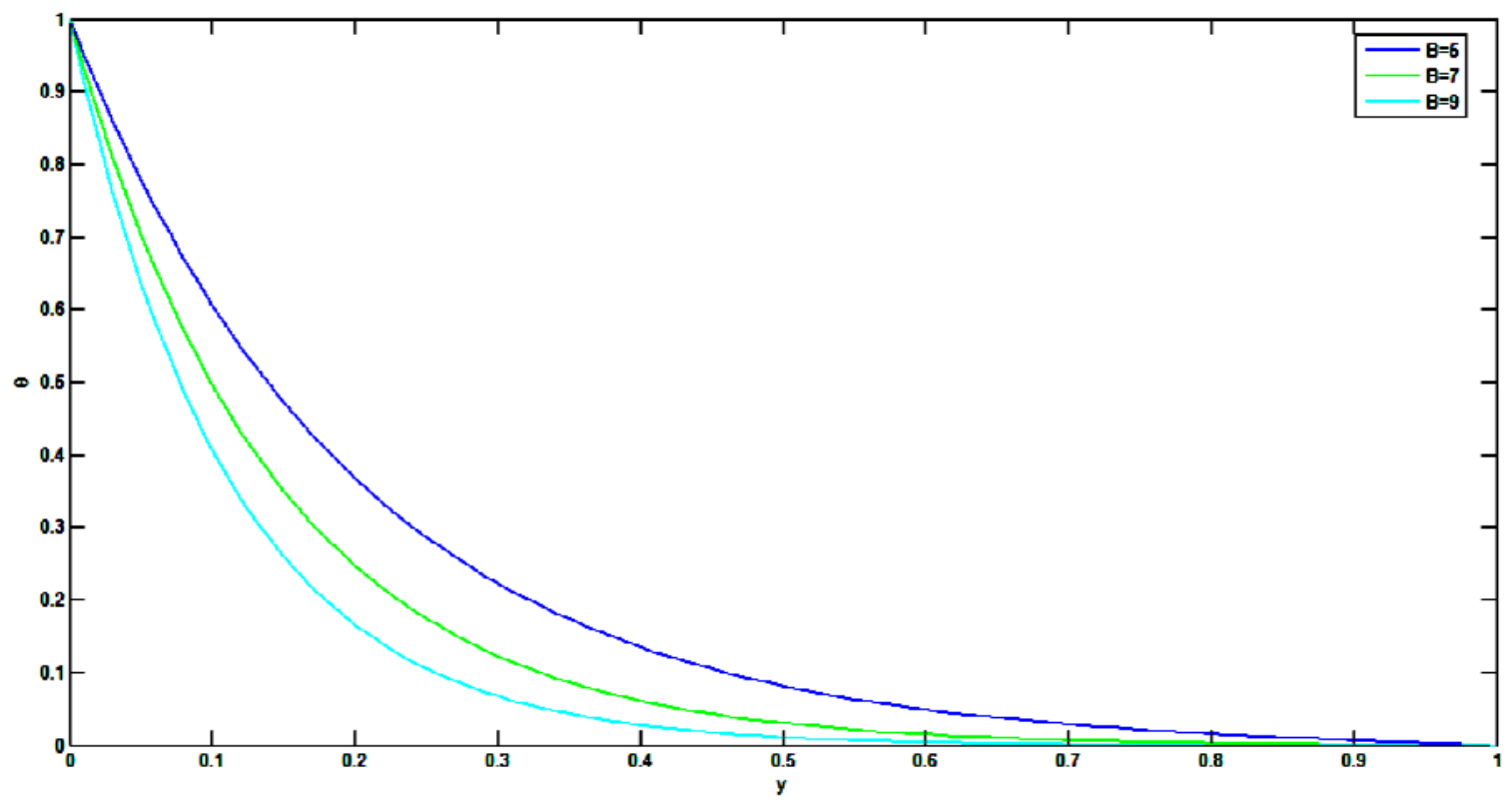

Fig.4. Temperature profile of the model for different values of strength of magnetic field.

In Fig.5 the simulated results of the influence of dynamic viscosity on the temperature profile have been presented. The graph is plotted yversus $\theta$ representing the distance between the channel walls and temperature, respectively. From the simulation study it can be observed that as the dynamic viscosity increases the temperature of the fluid decreases. The Prandtl number is directly proportional to dynamic viscosity and inversely proportional to thermal conductivity. This theoretical fact has been proved by the simulation study.

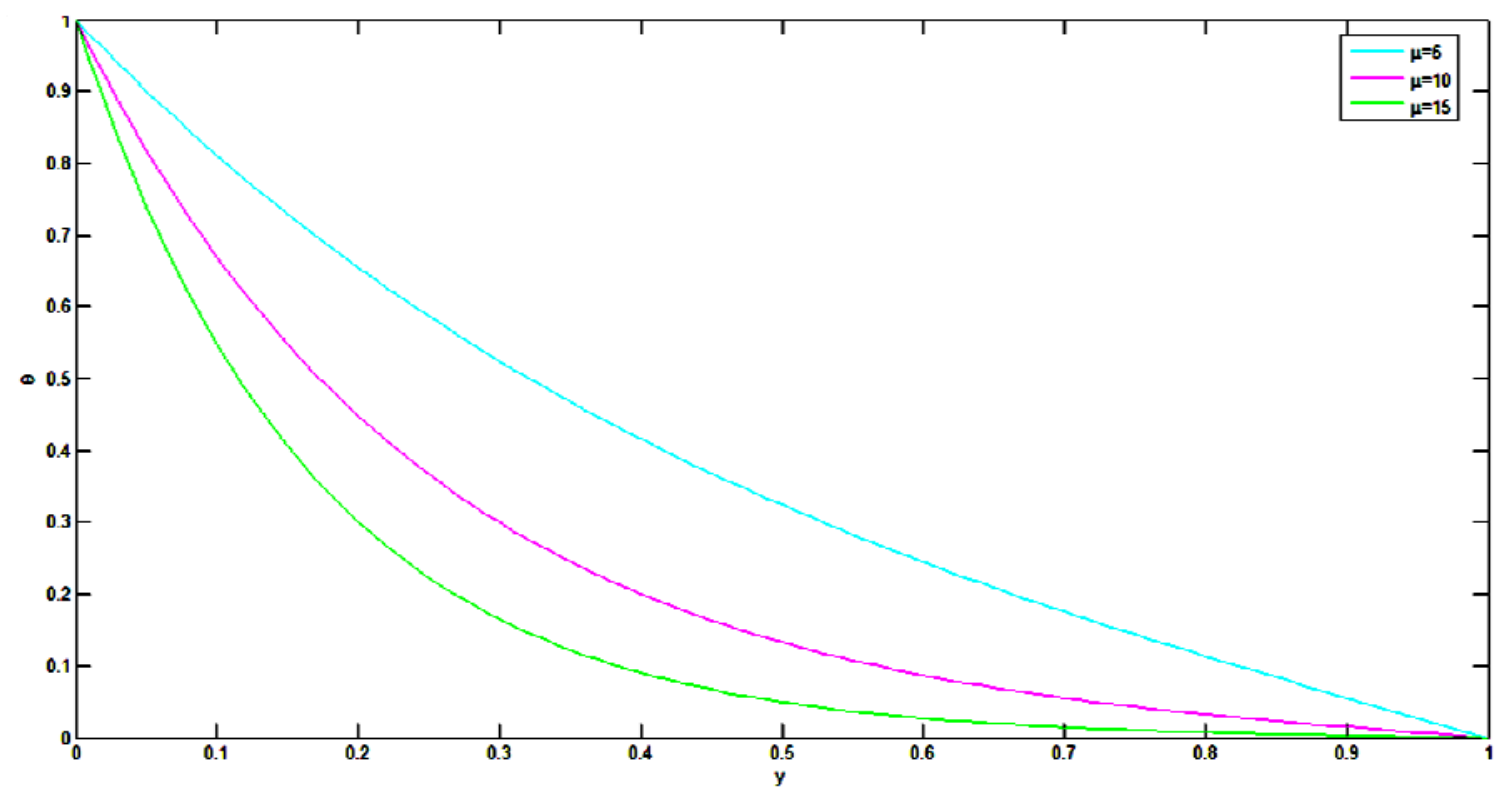

Fig.5. Temperature profile of the model for different values of dynamic viscosity. 


\section{Conclusion}

In this paper, thermal radiation, chemical reaction, viscous and Joule dissipation effects on MHD flow in a porous medium have been analyzed. The effects of various parameters, viz., the Eckert number, electric conductivity, dynamic viscosity and strength of magnetic field on the temperature profile have been studied.

From the simulation study the following results are obtained:

I. an increment of the non-dimensional quantity (Eckert number) results in a decrement of temperature profile;

II. as the values of electric conductivity increase the temperature profile of the fluid decreases.

III. increasing the values of strength of magnetic field results in a decrement of temperature.

VI. an increment of the values of dynamic viscosity results in a decrement of the temperature profile of the fluid.

\section{Nomenclature}

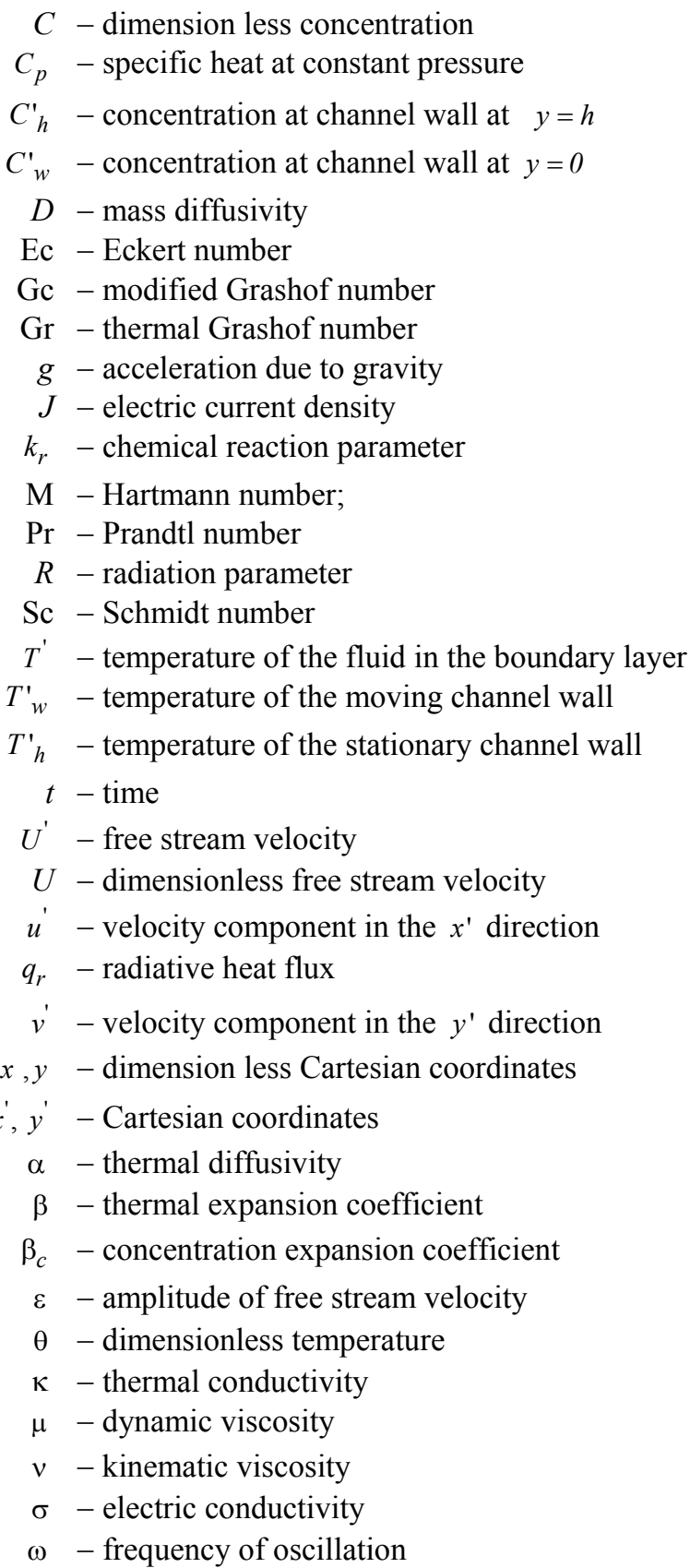




\section{References}

[1] Attia H.A. and Abdeen M.A.M. (2012): Unsteady MHD flow and heat transfer between parallel porous plates with exponential decaying pressure gradient. - Kragujevac J. Sci., vol.34, pp.15-22.

[2] Das S., Jana M. and Jana R.N. (2011): Couette flow through porous medium in a rotating system. - International Journal of Mathematical Archive, vol.2, No.11, pp.2318-2326.

[3] Attia H.A. and Ewis K.M. (2010): Unsteady MHD Couette flow with heat transfer of a viscoelastic fluid under exponential decaying pressure gradient. - Tamkang Journal of Science and Engineering, vol.13, No.4, pp.359-364.

[4] Chamkha A.J. (2002): On laminar hydromagnetic mixed convection flow in a vertical channel with symmetric and asymmetric wall heating conditions. - Int. J. Heat Mass Transfer, vol.45, pp.2509-2525.

[5] Singh A.K. (1988): Natural convection in unsteady Couette motion. - Def. Sci. J., vol.38, No.1, pp.35-41.

[6] Jha B.K. (2001): Natural convection in unsteady MHD Couette flow. - Heat and Mass Transfer, vol.37, pp.329-331.

[7] Rajput U.S. and Sahu P.K. (2012): Natural convection in unsteady hydromagnetic Couette flow through a vertical channel in the presence of thermal radiation. - Int. J. of Appl. Math and Mech., vol.8, No.3, pp.35-56.

[8] Job V.M. and Gunakala S. Rao (2013): Unsteady MHD free convection Couette flow through a vertical channel in the presence of thermal radiation with viscous and Joule dissipation effects using Galerkin finite element method. - International Journal of Application or Innovation in Engineering and Management, vol.2, No.9, pp.50-61.

[9] Zigta B. and Koya P.R. (2017): The effect of MHD on free convection with periodic temperature and concentration in the presence of thermal radiation and chemical reaction. - International Journal of Applied Mechanics and Engineering, vol.22, No.4, pp.1059-1073, DOI: 10.1515/ijame-2017-0068.

[10] Nield D.A. and Bejan A. (1998): Convection in Porous Media. - 2nd edition, Berlin: Springer Verlag.

[11] Singh A.K. (2002): MHD free convective flow through a porous medium between two vertical parallel plates. Ind. J. Pure and Appl. Phys., vol.40, pp.709-713.

[12] Helmy K.A. (1998): MHD unsteady free convective flow past a vertical porous plate. - Journal of Applied Mathematics and Mechanics, vol.78, pp.255-270.

[13] Hossain Md. Anwar and Pop I. (2001): Radiation effects on free convection over a vertical flat plate embedded in a porous medium with high porosity. - International Journal of Thermal Sciences, vol.40, pp.289-295.

[14] Ibrahim S.Y. and Makinde O.D. (2011): Radiation effect on chemically reacting MHD boundary layer flow of heat and mass transfer through a porous vertical flat plate. - International Journal of Physical Sciences, vol.6, pp.1508-1516.

[15] Manna S.S., Das S. and Jana R.N. (2012): Effects of radiation on unsteady MHD free convective flow past an oscillating vertical porous plate embedded in a porous medium with oscillatory heat flux. - Advance in Applied Science Research, vol.3, pp.3722-3736.

[16] Elbashbeshy E.M.A., Yassmin D.M. and Dalia A.A. (2010): Heat transfer over an unsteady porous stretching surface embedded in a porous medium with variable heat flux in the presence of heat source or sink. - African Journal of Mathematics and Computer Science Research, vol.3, No.5, pp.68-73.

[17] Angirasa D., Peterson G.P. and Pop I. (1997): Combined heat and mass transfer by natural convection with opposing buoyancy effects in a fluid saturated porous medium. - Int. J. Heat Mass Trans., vol.40, No.12, pp.2755-2773.

[18] Brewster M.A (1992): Thermal Radiative Transfer and Properties. - New York: John Wiley and Sons. 